\title{
Part 13: (e)Accessibility
}

\author{
Accessibility Equals Innovation \\ Vivienne Conway* and Amanda Mace \\ Web Key IT Pty Ltd, 10 Tonrita Place, Wanneroo, WA \\ 6065, Australia
}

Background: For people with disabilities, assistive technology (AT) is a necessity. AT is often prohibitively expensive for people with disabilities, especially new technology. If we can influence creators of AT of the opportunities for innovation through a Universal Design approach, the lives of people with disabilities will be improved, and appeal to a larger segment of users. While AT has largely come about through innovation to solve a particular need, that innovation has provided benefit for an unintended group of users. AT is largely seen as confined to people with disabilities, however, incorporating Universal Design principles broadens the commercial potential, making it more affordable. Developers should understand that meeting a particular user group's needs, provides an opportunity to benefit a larger user group. The role of Universal Design in AT, encourages design for a wider audience, encouraging innovation and enabling more economic solutions for accessibility issues.

Method used: We observe AT employed by our user testers, and see that what is essential for them, is now being used for different purposes. Technology such as eye-tracking and voice-activation software, was developed for people without the use of their hands to work with digital material. Eye-tracking is now being used to predict user behavior to determine the best placement of material. Voice activation, is being used by professionals, decreasing the time and cost of dictation/transcription. The principal of Universal Design shows that developing technology that will be usable by a broad group of users broadens the commercial potential for the technology, which may lower the cost and ensure better support than technology intended for more specific audiences.

Key results: Developing AT for people with disabilities has led to opportunities for innovation. Not only does the wheelchair ramp benefit someone in a wheelchair, it also benefits the mother pushing a pram. Global initiatives, such as the WHO's GATE program are looking to provide access to assistive technology for everyone, enabling the person to lead the type of life they wish. Embracing innovation which benefits the wider society, benefits more than people with disabilities. Incorporating the concept of Universal Design to create more accessible digital environments need not diminish the emphasis on the needs of people with disabilities. Enforcing accessibility meets with reluctance, though it is intended to make life better for people. If we were to change the motive for developing products to meet a wider possible audience, there would be greater commercial interest, and hence more innovation to assist users with disabilities.

Conclusion: AT is not a "nice to have, for a minority of people', it is a necessity for many. Incorporating Universal Design principles into AT development provides an opportunity to develop products that delight a wider audience, while also meeting the needs of people with disabilities. The practical implication is that if we change our mindset about the audience for technology advancement for people disabilities, we have an added commercial incentive to sell products that improve lives for a wide range of people.

${ }^{*}$ Corresponding author. E-mail: v.conway@webkeyit. com

\section{Complex PDF Remediation for Accessibility: Re- view of Current Methodologies}

Valentín Salinas López

Institut Integriert Studieren, Johannes Kepler Universität Linz, Altenberger Straße 69, 4040 Linz, Austria

Background: There is a growing demand to remediate complex non-accessible PDF. For instance, schoolbooks which are required to be accessible and show a wide variability in their morphology. A problem lies in the existence of an extensive bibliography of digi- 
tal teaching material that are delivered using PDF not accessible. Currently the tasks involved in making a PDF accessible are expensive due that many of them have to be performed manually. Among these, structuring PDF content is especially time-consuming. Tagging i.e. associating a label to each significant element of the document, is a prime example thereof, as it is of uttermost importance in order to enable assistive technologies, such as screen readers or accessible forms for motor disabled people that do not use analog pointing devices. The question that arises is whether it is possible to make this process cheaper. To do so this tries to identify the current needs in the remediation procedure to enable a following research about improvements.

Method: In a first stage, a comparison is made between the current PDF reading and authoring tools. The selection criteria for the software to be compared is, its popularity and it must be intended for an individual user using a PC. This filter keeps out little-known software that professional of the document accessibility remediation seldom use, and also online remediation services that often not to allow public access to their internal procedures.

In second stage, it is chosen one tool. With this, it is analyzed the procedure to make PDFs accessible. The effort is measured using the amount of steps, the number of user interactions to achieve each step and the obstacles and inconsistencies found in the workflow.

The documents used in the second stage are nonaccessible PDF schoolbooks. Since these meet the requirements of complexity and need for remediation.

Key results: Acrobat Reader DC is chosen after analyzing the comparative. The main reasons are that Acrobat is de facto desktop application for working with PDFs, other software describe a procedure for remediation quite similar or need the use of Acrobat in some steps. It worth mention that many analyzed tools are not able to accomplish completely the procedure of making accessible PDFs.

Results show that Acrobat is not optimized for accessibility remediation. The user has to perform complex manual actions very often. Automations offered by the software are useful and save time and effort, but fail when the complexity of the document increases slightly. Many times, correct the results of the automations involve doing greater effort actions than the necessary ones to accomplish procedure in a completely manual manner.

Conclusion: The process of fix the accessibility of PDFs can be improved using a tool designed specifically for this purpose. Acrobat DC may be a good choice for the remediation of documents with simple structure, but not in other case mainly because poor results of the automated task. Therefore, there is room for future improvements in the automation of tasks for the treatment of complex documents.

Keywords: PDF, Accessibility remediation, SOTA, tool comparison, teaching material.

${ }^{*}$ Corresponding author. E-mail: valentin.salinas_lopez @jku.at

Holistic Evaluation Method and Tools for Local Government Websites Case Study: EEC, Thailand Prajaks Jitngernmadan*

Faculty of Informatics, Burapha University, 169 Longhard Bangsaen Rd., Saensook, Muang, Chon Buri, 20131, Thailand

Background: Eastern Economic Corridor (EEC) locates in the Eastern part of Thailand, which should facilitate the Thai economy in the long run. Thai government sets this area to be the heart of national innovation and technology development. While the economic aspect is the main target, the social aspect is also important due to the great number of estimated migrant workers (more than 33,000). Currently, local governments set up their own websites providing information and e-government services; including relocation, registration, ID card extending, etc.

Since the variety of the potential users is tremendous, these websites have to universally usable and accessible. There are works tried to evaluate Thai government websites. However, they only covered evaluation partially, without consideration of social and economic aspects. The aim is to identify factors that affect the accessibility, usability, and user experience and combining each factor and its testing method into an areabased holistic evaluation method, which covers 4 areas including Availability, Mobile Device Readiness, Accessibility, and Usability.

Method: The most important 10 local governments' websites are selected for evaluation in our laboratory. The availability is defined through a web content download speed over slow Internet connection, reachability upon multiple requests, web content display ability, and security issue. For testing, a semi-automatic testing toolset was chosen: the Google Chrome Development Tools. These tools are used for different Internet connection speed simulation, secure protocol HTTPS, and meaningful page load checking. The mobile device readiness is defined through meaningful re- 
sponsive web design for small displays. The Google Chrome Development toolset is used to simulate a website in different screen sizes. The accessibility of a website is tested using the W3C Markup Validator and WAVE from WebAIM. The first one is used to check HTML implementation. The latter is used for accessibility testing against WCAG 2.0 level AA. Furthermore, a color contrast between foreground and background colors is examined. The standardized heuristic evaluation for user interface design, suggested by Jakob Nielsen, is used for usability testing and it contains 10 usability criteria, which is evaluated by experts.

Key results: In terms of availability, only 3 out of 10 websites passed the speed test, and none of them uses secure protocol. For mobile device readiness, 3 websites can be considered as ready due to their meaningful responsive design. For accessibility, only 4 websites have errors less than 50. Most of them have a high number of programming syntax errors. And for usability, none of the websites can reach the highest score. Furthermore, $60 \%$ of the websites use Flash content only for decorative purpose.

Conclusion: For evaluating local governments' websites by means of supporting economic and social aspect, different factors and evaluation strategies have to be identified and tested. We suggest 4 evaluating aspects (Availability, Mobile Device Readiness, Accessibility, and Usability) for local governments in EEC area. The combination of evaluation factors and methods can be used as a standard strategy for evaluation. The next step is to provide a handy toolset and manual for standardized Thai local governments' website evaluation.

Keywords: Website, Accessibility, Holistic, Government, Evaluation.

*Corresponding author. E-mail: prajaks@buu.ac.th

Usability Assessment of an Accessible Voting System - A Mixed Method Study

Joaquim Alvarelhão ${ }^{a}$, Nelson P. Rocha ${ }^{b, *}$

${ }^{a}$ School of Health Sciences, IEETA, University of Aveiro, Campo Universitário de Santiago, 3810-193 Aveiro, Portugal

${ }^{b}$ Department of Medical Sciences, IEETA, University of Aveiro, Campo Universitário de Santiago, 3810-193 Aveiro, Portugal

Background: Persons with disability have fewer opportunities to participate in politics, including voting in elections. There is a lack of information about the participation levels of persons with limitations in fulfilling the ballot in paper. Although in some countries it may be possible to use alternative forms, such as voting accompanied by another person, there is still no system to guarantee the person's independence in completing the ballot paper in a secret manner. This experimental study aimed to evaluate the usability from the point of view of the user of an Accessible Voting System (AVS) in a real election.

Method: A parallel mixed method study was conducted in the Executive Board election of Oporto's Cerebral Palsy Association, Portugal. From the seventy total voters, thirty-one adults (twelve with Cerebral Palsy, GMFCS: I-II = 5; III-V = 7) that choose to vote using the AVS were included (19 males, aged between 24-73 years old). The AVS comprises: (i) a computer application with voting options; (ii) visual and auditory instructions for the user; (iii) different input methods like touchscreen or scanning; (iv) different simultaneous output methods such visual or auditory; and (v) a printer for produce an individual secret ballot paper. A quantitative usability assessment was made using Post Study System Usability Questionnaire (PSSUQ) that comprises 19 items (1-7 Likert scale, $1=$ better usability). In addition to a global score, PSSUQ provides results in three domains: (i) System Usefulness; (ii) Information Quality; and (iii) Interface Quality. Qualitative data of persons with Cerebral Palsy was obtained through a structured interview covering: (i) previous experiences of voting; (ii) what was the sensation of using the system; and (iii) recommendations for improvement.

Key results: Global usability score for PSSUQ was mean $=1.3(\mathrm{dp}=0.35)$. For subscales System Usefulness, Information Quality and Interface Quality scores were mean $=1.1(\mathrm{dp}=0.30)$, mean $=1.2(\mathrm{dp}=$ $0.55)$, mean $=1.5(\mathrm{dp}=0.80)$, respectively. No difference was found in scores between Cerebral Palsy voters and other users. In the same sense, no association was found between PSSUQ results and age or gender. From users with Cerebral Palsy, two never had voted before, eight used to vote accompanied by another person and two by themselves. The sensation of using the system was described as: [A] "System is accessible and practical in use."; [B] "More autonomy."; [C] "Easier than manual voting."; [D] "[...] because I went alone, it was good do not have to take anyone with me, it should be implemented at the national level [...]."; [E] "I felt it was easy to understand."; [F] "A freedom, so the vote is 'more' secret, nobody knows. 
Must be this way to vote for the Government."; [G] "I felt I was doing a normal act of citizenship. I think it should be implemented all over the country."; [H] "It was my first secret ballot [...]."; [I] "I feel very well and comfortable, it's very easy.".

Conclusion: The results shown a high level of usability of the AVS and can contribute for this kind of tools to be made available in national and European elections. Other more in-depth studies should be carried out, including in other populations, in order to confirm the results.

Keywords: Accessible voting system, Usability assessment, Mixed method assessment.

*Corresponding author. E-mail: npr@ua.pt 